\title{
Common Adult Vaccinations May Reduce Risk for Dementia
}

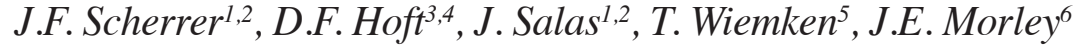

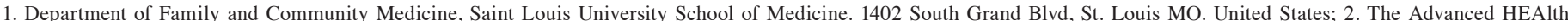

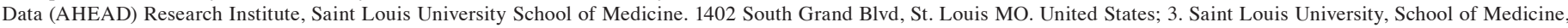

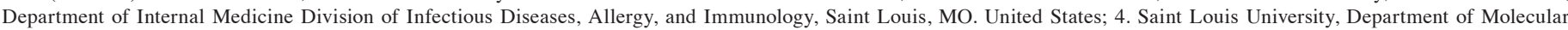

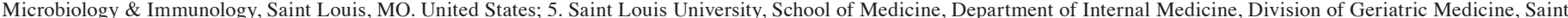

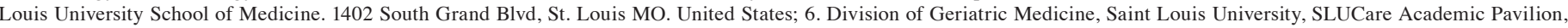 \\ Section 2500, 1008 S. Spring Ave.,St. Louis, MO, United States
}

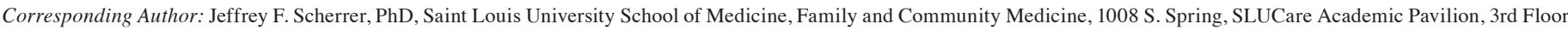
St. Louis, MO 63110, scherrjf@slu.edu, voice:314-977-8486

$7 \mathrm{t}$ is estimated that 55 million people in the world have dementia and it is expected that 10 million new cases will occur annually (1). Currently, there are no treatments that can substantially reduce the risk for dementia. However, a recent series of studies now indicate that common adult vaccinations may be a low-cost, accessible means to reduce dementia risk by 20 to $60 \%(2-7)$. The logic behind vaccinations as a potential means to reduce dementia risk is based on evidence that viral, bacterial, and fungal infections contribute to increased risk for dementia (8), and evidence that infection plays a key role in the etiology of Alzheimer's Disease (AD) $(9,10)$.

If infections contribute to dementias, then logically, those who are vaccinated against these threats should have a lower risk vs. those not vaccinated. Indeed, in the Canadian Study of Health and Aging, participants who self-reported prior diptheria, polio or tetanus vaccination compared to those who did not report receipt of these vaccinations had a $60 \%$ lower AD incidence even after controlling for family history of dementia (2). In three separate retrospective cohort studies (3, $4,7)$ receipt of influenza vaccination was associated with lower risk for dementia and a greater number of annual influenza vaccinations was associated with greater reduction in risk $(3,7)$.

Across multiple vaccine types, we have recently found a reduced risk for dementia in persons who receive vs. do not receive specific vaccinations. We used similar methods and analytic approaches for each vaccine type studied. De-identified data from 2008 - 2019 were obtained from large cohorts $(>100,000$ patients $)$ created from Veterans Health Administration (VHA) and MarketScan medical claims. Eligible patients were all 65 years or older at baseline. In each study, we required patients to be free of dementias and conditions associated with cognitive decline prior to baseline. We controlled for confounding using propensity scores or entropy balancing and weighted data to remove differences in the distribution of covariates across vaccine status. For instance, use of well visits might be associated with better overall health, reduced risk for dementia and greater likelihood of obtaining appropriate vaccinations. This variable and many other covariates including demographics, geographic region, health services use, medication use, and physical and psychiatric comorbidities were controlled for by using propensity scores and inverse probability of treatment weighting to balance their distribution between vaccinated and unvaccinated patients. Following this approach, we observed that patients who received tetanus, diphtheria and pertussis (Tdap) vaccination, compared to those who remained without Tdap, were $42 \%$ less likely to develop dementia in both VHA and MarketScan claims data (5).

Our next study focused on influenza vaccination and incident dementia. Compared to VHA patients, those with private health care insurance may be more likely to obtain influenza vaccinations from sources other than their physician (e.g. health fairs, employer). Therefore, our study of influenza vaccination was limited to VHA patients which reduced misclassification of influenza vaccination. The magnitude of association between influenza vaccination and incident dementia was less that that observed with other types of vaccines $(\mathrm{HR}=0.86$; 95\%CI:0.83-0.88) (7). Even in a VHA cohort, misclassifying influenza vaccination is more likely than misclassifying Tdap vaccination. Such misclassification would tend to bias our estimates to the null.

While these two studies are consistent with existing literature, we were not able to account for exposure to infections with tetanus, diphtheria and pertussis, or the contribution of influenza to dementia. Because the former infections are so rare and most cases of influenza do not involve medical care and are not documented in the medical record or claims data, we could not be certain if the association was due to vaccination protecting against these infections and subsequent dementia or explained by some type of non-specific vaccination effect.

Subsequently, we investigated the association between herpes zoster (HZ) vaccination and incident dementia. Following similar methods, we observed that $\mathrm{HZ}$ vaccination, compared to remaining without $\mathrm{HZ}$ vaccination, was associated with lower risk for dementia in VHA $(\mathrm{HR}=0.69$; 95\%CI:0.670.72) and MarketScan (HR=0.65; 95\%CI:0.57-0.74) data (6). Importantly, the relationship between $\mathrm{HZ}$ vaccination and dementia did not change after accounting for the contribution from $\mathrm{HZ}$ reactivation and use of antivirals between index and end of follow-up (6). This suggests that $\mathrm{HZ}$ vaccination did not prevent $\mathrm{HZ}$ infections that led to subsequent dementia, but instead, HZ vaccination had a non-specific effect in reducing 
dementia risk. Of note, because we had access to mortality data in the VHA, we were able to compute competing risk models to account for bias related to mortality.

There are several potential explanations for the relationship between vaccination and dementia. First, those with higher education and income are more likely to receive appropriate adult vaccinations and less likely to develop dementia. However, we controlled for zip code level socioeconomic status in our work and found no change in results. In addition, we replicated findings from VHA data in MarketScan medical claims. VHA patients overall have a lower socioeconomic status compared to those with private insurance which further reduces the chance that our results are explained by income or education. Another explanation could be the healthy adherer effect. Specifically, those who obtain vaccinations also adhere to other medical regimens that reduce risk for dementia. In sensitivity analyses, adjusting for adherence to anti-hypertensive medications had no effect on the association between vaccination and dementia (under review). Thus, the evidence points to an association between vaccination and dementia, independent of patient socioeconomic status and medical regimen adherence.

It remains possible that vaccinations have protective effects either against the specific pathogen targeted for prevention by the vaccine, or more broadly protective effects against pathogens that cause neuroinflammation leading to increased risks of incident dementia. However, given the lower risk for dementia across a wide range of vaccine types and the consistency of our hazard ratios after accounting for $\mathrm{HZ}$ infection following $\mathrm{HZ}$ vaccination, the weight of evidence does not support a specific vaccine effect directed against the vaccine targeted pathogens. Instead, we speculate that vaccinations might train the immune system to respond to viral, bacterial or noninfectious inflammatory threats and prevent neuroanatomical damage. By training the immune system to be more tightly regulated, routine vaccinations may reduce inflammation and oxidative stress which contribute to the etiology of dementia.
The existing literature is not sufficient to conclude a causal relationship between vaccination and dementia. Long-term, prospective cohort studies are needed to confirm our results. An important future direction should determine if vaccination might slow or prevent transition from mild cognitive impairment to dementia. Finally, the effect of vaccination on memory tasks in rodent models of dementia could confirm a causal role for vaccines in reducing dementia, and reveal the mechanisms responsible for our current theory of vaccinationinduced immune training effects leading to decreased neuroinflammation as a means to reduce dementia.

Conflicts of interest: Authors report no conflict of interest.

Funding: The authors' research studies summarized in this article were funded by the Benter Foundation, grant 2020-01.

\section{References}

1. Alzheimer's Association Report. 2020 Alzheimer's disease facts and figures. Alzheimer's and Dementia 2020;16:391-460.

2. Verreault R, Laurin D, Lindsay J, Serres GD. Past exposure to vaccines an subsequent risk of Alzheimer's disease. CMAJ: Canadian Medical Association Journal 2001;165:1495.

3. Luo CS, Chi CC, Fang YA, Liu JC, Lee KY. Influenza vaccination reduces dementia in patients with chronic obstructive pulmonary disease: a nationwide cohort study. J Investig Med 2020;68:838-45.

4. Liu JC, Hsu YP, Kao PF, et al. Influenza Vaccination Reduces Dementia Risk in Chronic Kidney Disease Patients: A Population-Based Cohort Study. Medicine (Baltimore) 2016;95:e2868.

5. Scherrer JF, Salas J, Wiemken TL, Jacobs C, Morley JE, Hoft DF. Lower Risk for Dementia Following Adult Tetanus, Diphtheria and Pertussis (Tdap) Vaccination. J Gerontol A Biol Sci Med Sci 2021;76:1436-43.

6. Scherrer JF, Salas J, Wiemken TL, Hoft DL, Jacobs C, Morley JE. Impact of Herpes Zoster Vaccination on Incident Dementia: A Retrospective Study in Two Patient Cohorts PLoS One In Press.

7. Wiemken TL, Salas J, Hoft DF, Jacobs C, Morley JE, Scherrer JF. Dementia risk following influenza vaccination in a large veteran cohort running head: Influenza vaccination and dementia. Vaccine 2021.

8. Mawanda F, Wallace R. Can infections cause Alzheimer's disease? Epidemiol Rev 2013;35:161-80

9. Abbott A. Are infections seeding some cases of Alzheimer's disease? Nature 2020;587:22-5

10. Sochocka M, Zwolinska K, Leszek J. The Infectious Etiology of Alzheimer's Disease. Curr Neuropharmacol 2017;15:996-1009.

How to cite this article: J.F. Scherrer, D.F. Hoft, J. Salas, et al. Common Adult Vaccinations May Reduce Risk for Dementia. J Nutr Health Aging. 2021;25(10):11381139, http://dx.doi.org/10.1007/s12603-021-1695-2 